


\section{Verlag von Veit \& Comp. in Leipzig}

\section{DAS STEREOSKOP.}

Seine Anwendung in den technischen Wissenschaften.

Über Entstehng and Konstruktton stereoskoplscher Bilder.

Von

\section{Professor Wilhelm Manchot.}

Mit 50 Figuren.

gr. 8. 1903. geh. I M. 80 Pf.

\section{ELEMENTARE MECHANIK}

als Einleitung in das Studium der theoretisehen Physik. Von

Dr. Woldemar Voigt,

o. ö. Professor der Physik an der Universität Göttingen.

Zweite, umgearbeitete Auflage.

Mit 56 Figuren im Text. Lex. 8. 1901. geh. 14 M., geb. in Halbfranz 16 M.

\section{DIE MUTATIONSTHEORIE.}

Versuche und Beobachtungen

über die Entstehung von Arten im Pflanzenreich. Vैon

Hugo de Vries,

Professor der Botanik in Amsterdam.

Zwei Bände.

Roy. 8. geh. 43 M., geb. in Halbfranz 49 M.

Erster Band. Die Entstehung der Arten durch Mutation. Mit zahlreichen Abbildungen im Text und acht farbigen Tafeln. 1901. geh. 20 M., geb. in Halbfranz $23 \mathrm{M}$.

Zweiter Band. Elementare Bastardlehre. Mit zahlreichen Abbildungen im Text und vier farbigen Tafeln. 1903. geh. $23 \mathrm{M}$, geb. in Halbfranz $26 \mathrm{M}$.

Gestützt auf eine lange Reihe ausgezeichneter Untersuchungen und auf ausgedehnte Literaturstudien liefert der Verfasser in diesem epochemachenden Werke ein ungemein reiches Material zur Entscheidung der Frage, wie neue Arten entstehen. Der Darwinismus beantwortet diese Frage bekanntlich dahin, daB Arten ganz allmählich aus anderen hervorgehen, de Vries weist dagegen nach, daB die "fluktuierende Variation", auf welche sich der Darwinismus fast ausschlieblich stützt, zur Bildung neuer Arten nicht führen kann. Neue Arten entstehen stoßweise. Diese Stöße nennt de Vries „Mutationen“. Er zeigt, daß diese Entstehung sich ebenso gut beobachten läßt, wie jeder andere physiologische Vorgang. 
Verlag von VEIT \& COMP. in Leipzig.

DRAHTLOSE TELEGRAPHIE DURCH WASSER UND LUFT.

Nach Vorträgen, gehalten im Winter 1900

von

Prof. Dr. Ferdinand Braun,

Direktor des Physikalischen Institutes der Universität Straßburg.

Mit zahlreichen Figuren und Abbildungen.

Lex. 8 1901. geh. 2 M.

\section{DIE \\ PHYSIOLOGISCHEN FERNWIRKUNGEN DER ELEKTRIZITÄT.}

Untersuchungen

von

Dr. Basile Danilewsky,

Professor der Physiologie an der Universität zu Charkow.

Mit zahlreichen Abbildungen. - gr. 8. 1902. geh. 7 M.

\section{DAS RADIUM.}

SEINE DARSTELLUNG UND SEINE BigeNSCHAFTEN.

$$
\text { Von }
$$

\section{Dr. Jacques Danne,}

Privatassistenten des Herrn Professor Pierre Curie.

Mit einem Vorwort von Charles Lauth, Direktor der Hochschule für angewandte Physik und Chemie zu Paris.

Mit zahlreichen Figuren.

Autorisierte Ausgabe. 8. I904. kart. 2 M. 40 Pf.

\section{ELEKTROCHEMIE.}

I HRE GESCHICHTE UND LEHRE.

$$
\text { Von }
$$

\section{Wilhelm Ostwald.}

Mit 260 Nachbildungen geschichtlicher Originalfiguren.

Lex 8. 1896. geh. $28 \mathrm{M}$, eleg. geb. 30 M. 
Verlag von VEIT \& COMP. in Leipzig.

\title{
ÜBER DIE GRENZEN DES NATURERKENNENS. DIE SIEBEN WELTRÄTSEL.
}

\author{
Zwei Vorträge \\ von \\ Emil du Bois-Reymond.
}

Des ersten Vortrages neunte, der zwei Vorträge fünfte Auflage.

8. 1903. geh. 2 M.

\section{DIE ENERGETIK}

NACH IHRER GESCHICHTLICHEN ENTWICKELUNG.

Von

Dr. Georg Helm,

o. Professor an der Königl. Technischen Hochschule zu Dresden.

Mit Figuren im Text.

gr. 8. 1898 . geh. 8 M. 60 Pf., geb. in Ganzleinen 9 M. 60 Pf.

\section{ABHANDLUNGEN UND VORTRÄGE ALLGEMEINEN INHALTS}

\author{
(1887--1903). \\ Von
}

Wilhelm Ostwald.

gr. 8. 19.94. geh. 8 M., geb. in Ganzleinen 9 M.

\section{VORLESUNGEN ÜBER \\ THER M OD Y N A M IK \\ von}

Max Planck.

Mit fünf Figuren im Text.

gr. 8. 1897. kart. in Ganzleinen 7 M. 50 Pf. 


\title{
DIE \\ DRAHTLOSE TELEGRAPHIE
}

\author{
AUF GRUND \\ EIGNER PRAKTISCHER ERFAHRUNGEN \\ voN
}

DR. PHIL. GUSTAV EICHHORN

MIT ZAHLREICHEN FIGUREN.

VERLAG VON VEIT \& COMP. IN LEIPZIG

1904 
Druck von Fischer \& Wittig in Leipzig. 


\section{Meiner lieben Mutter}

$$
\text { gewidmet }
$$


